\title{
Multiple association analysis of loci and candidate genes that regulate body size at three growth stages in Simmental beef cattle
}

Bingxing $\mathrm{An}^{\dagger}{ }^{\dagger}$, Lei $\mathrm{Xu}^{\dagger}$, Jiangwei Xia ${ }^{2}$, Xiaoqiao Wang ${ }^{1}$, Jian Miao ${ }^{1}$, Tianpeng Chang ${ }^{1}$, Meihua Song ${ }^{3}$, Junqing $\mathrm{Ni}^{4}$, Lingyang $\mathrm{Xu}^{1}$, Lupei Zhang ${ }^{1}$, Junya $\mathrm{Li}^{1}$ and Huijiang Gao ${ }^{1 *}$ (D)

\begin{abstract}
Background: Body size traits as one of the main breeding selection criteria was widely used to monitor cattle growth and to evaluate the selection response. In this study, body size was defined as body height (BH), body length (BL), hip height $(\mathrm{HH})$, heart size $(\mathrm{HS})$, abdominal size (AS), and cannon bone size (CS). We performed genome-wide association studies (GWAS) of these traits over the course of three growth stages $(6,12$ and 18 months after birth) using three statistical models, single-trait GWAS, multi-trait GWAS and LONG-GWAS. The Illumina Bovine HD 770 K BeadChip was used to identify genomic single nucleotide polymorphisms (SNPS) in 1217 individuals.

Results: In total, 19, 29, and 10 significant SNPs were identified by the three models, respectively. Among these, 21 genes were promising candidate genes, including SOX2, SNRPD1, RASGEF1B, EFNA5, PTBP1, SNX9, SV2C, PKDCC, SYNDIG1, AKR1E2, and PRIM2 identified by single-trait analysis; SLC37A1, LAP3, PCDH7, MANEA, and LHCGR identified by multi-trait analysis; and P2RY1, MPZL1, LINGO2, CMIP, and WSCD1 identified by LONG-GWAS.

Conclusions: Multiple association analysis was performed for six growth traits at each growth stage. These findings offer valuable insights for the further investigation of potential genetic mechanism of growth traits in Simmental beef cattle.
\end{abstract}

Keywords: Simmental beef cattle, Genome-wide association studies, Body size, Candidate genes, Bovine HD 770 K SNP

\section{Background}

In China, the production of beef cattle is a very important agribusiness, and the Simmental breed accounts for more than $70 \%$ of beef-producing herds. Beef producers use body size to monitor the growth of each animal throughout the fattening period [1,2], as this trait is an indicator of cattle [3] and longevity [4]. Monitoring the development of each animal can help to increase profits by

\footnotetext{
*Correspondence: gaohuijiang@caas.cn

${ }^{+}$Bingxing An and Lei Xu contributed equally to this work.

'Institute of Animal Science, Chinese Academy of Agricultural Science, Beijing 100193, China

Full list of author information is available at the end of the article
}

enhancing the efficiency of feed and management [5-7]. Besides in human, additive genetic effect explains $81 \%$ of the variation in height [8], and the heritability for both hip height $(\mathrm{HH})$ and height size (HS) is $0.33-0.4$ in cattle [9]. Bouwman et al. reported that the lead variants in significant regions explained at most $13.8 \%$ of the phenotypic variance in their meta-analysis of 58,265 cattle from 17 populations [10]. In addition, daily body linear measurements, specifically body height $(\mathrm{BH})$ and $\mathrm{HH}$, two highly reliable and accurate indicators for body weight, are easier to obtained than body weight [11]. Furthermore, good depth of HS in cattle is a sign of good feet and leg

(c) The Author(s). 2020 Open Access This article is licensed under a Creative Commons Attribution 4.0 International License, which permits use, sharing, adaptation, distribution and reproduction in any medium or format, as long as you give appropriate credit to the original author(s) and the source, provide a link to the Creative Commons licence, and indicate if changes were made. The images or other third party material in this article are included in the article's Creative Commons licence, unless indicated otherwise in a credit line to the material. If material is not included in the article's Creative Commons licence and your intended use is not permitted by statutory regulation or exceeds the permitted use, you will need to obtain permission directly from the copyright holder. To view a copy of this licence, visit http://creativecommons.org/licenses/by/4.0/ The Creative Commons Public Domain Dedication waiver (http://creativecommons.org/publicdomain/zero/1.0/) applies to the data made available in this article, unless otherwise stated in a credit line to the data. 
conformation [12], and dairy cows with higher $\mathrm{HH}$ will subsequently have better milk performance [13]. However, there is little information on the molecular mechanisms of body size traits in Chinese Simmental beef cattle.

Genome-wide association studies (GWAS) are robust statistical tools are that broadly identify candidate genes with significant SNPs involved in production traits [14$16]$, growth traits $[17,18]$, carcass quality traits and fertility traits $[19,20]$. In beef cattle, various SNPs, genes, and haplotype blocks have been found to associate with growth, however the current GWAS-based studies focus mainly on only one growth parameter [21], such as the weaning size [22], yearling weight or stature upon slaughter [23]. Furthermore, loci controlling growth traits may be variable in different growth stages, and some loci may control traits throughout the lifetime of the animal [24]. Therefore, it is more reasonable to perform GWAS on growth traits on each stage separately. Multi-trait methods have been developed to increase statistical power and to identify pleiotropic loci in GWAS [25]. The longitudinal GWAS consider all time points when assessing whether significant SNPs associate with trait development over time [26], and this method is powerful for identifying these time-dependent and consistent loci [27]. We performed multiple trait GWAS (multi-trait GWAS) and longitudinal-GWAS (LONGGWAS) based on single-trait analysis. Multi-trait GWAS and LONG-GWAS were not replacements for the single-trait GWAS; instead, they complemented singletrait GWAS. Thus, understanding the genetic mechanisms involved in inter-individual variations in body size may provide new insights that can help to manipulate cattle growth and production.

In this study, six body size traits were routine measured from the time cattle entering farm to slaughter, which provides valuable resources to study the complete growing period. The aim of our study was to comprehensively analyze of candidate genes and QTL regions associated with growth traits by conducting three GWAS approaches in Simmental beef cattle. Our findings offer valuable insights for the further investigation of the potential mechanism of growth traits in Simmental beef cattle.

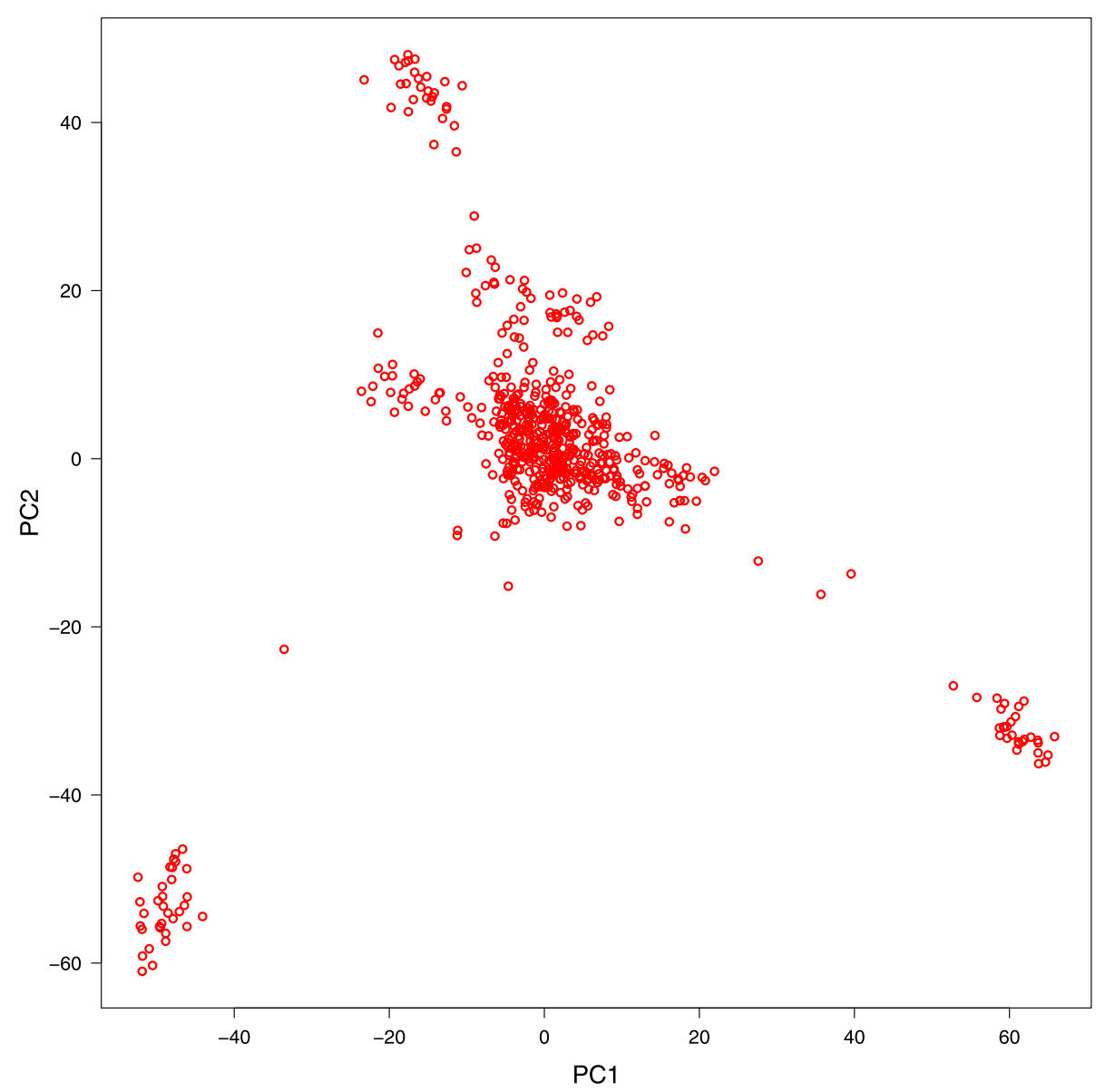

Fig. 1 Principal components (PC) plot drawn from the second principal component (PC2) against the first principal component (PC1) 


\section{Results}

\section{Population stratification assessment}

Figure 1 shows that the population stratification of the Simmental population based on the PCA was divided into five separate clusters, demonstrating an obvious stratification in the reference population. The population stratification caused by different genetic influences and breeding conditions, as potential confounders, was corrected by significance testing. We summarized the genome-wide significant and suggestive SNP regions for these traits in Fig. 2. The Manhattan plots and quantile-quantile (Q-Q) plots are shown in Figure S1 and Figure S2, while Q-Q plots suggested that there was no inflation or systematic bias in this research. Most points were near diagonal line because the GWAS model sufficiently considered the population structure and only a few SNPs were associated with the target traits. Meanwhile, the genomic inflation factors $(\lambda)$ at each trait ranged from 1.03 to 1.10 , indicating consistent consequence with PCA.

\section{Summary of significant loci identified by three approaches}

Briefly, we found 45, 66 and 19 SNPs significantly were associated with six body size traits by single-trait GWAS, multi-trait GWAS and LONG-GWAS, respectively. There were no significant loci for single-trait BH6 (single-trait GWAS for $\mathrm{BH}$ at 6 months after birth, and so forth), single-trait AS6, single-trait CS6, and LONG-AS. In addition, ten SNPs were associated with at least one of the six traits and eight SNPs were strongly associated with these traits in at least one of the three models. While according to their biological functions, 21 suggestive genes were selected as candidate genes and some details of them, including their positions in the genome, the nearest reported genes, the minor allele frequencies (MAF) and the $p$ values are listed in Table 1 .

\section{SNPs identified by single-trait GWAS}

A total of 45 SNPs achieved genome-wide significance associated with at least one of the six traits, with the $p$-value ranging from $9.99 \times 10^{-6}$ (BovineHD0700018941 for BL18) to

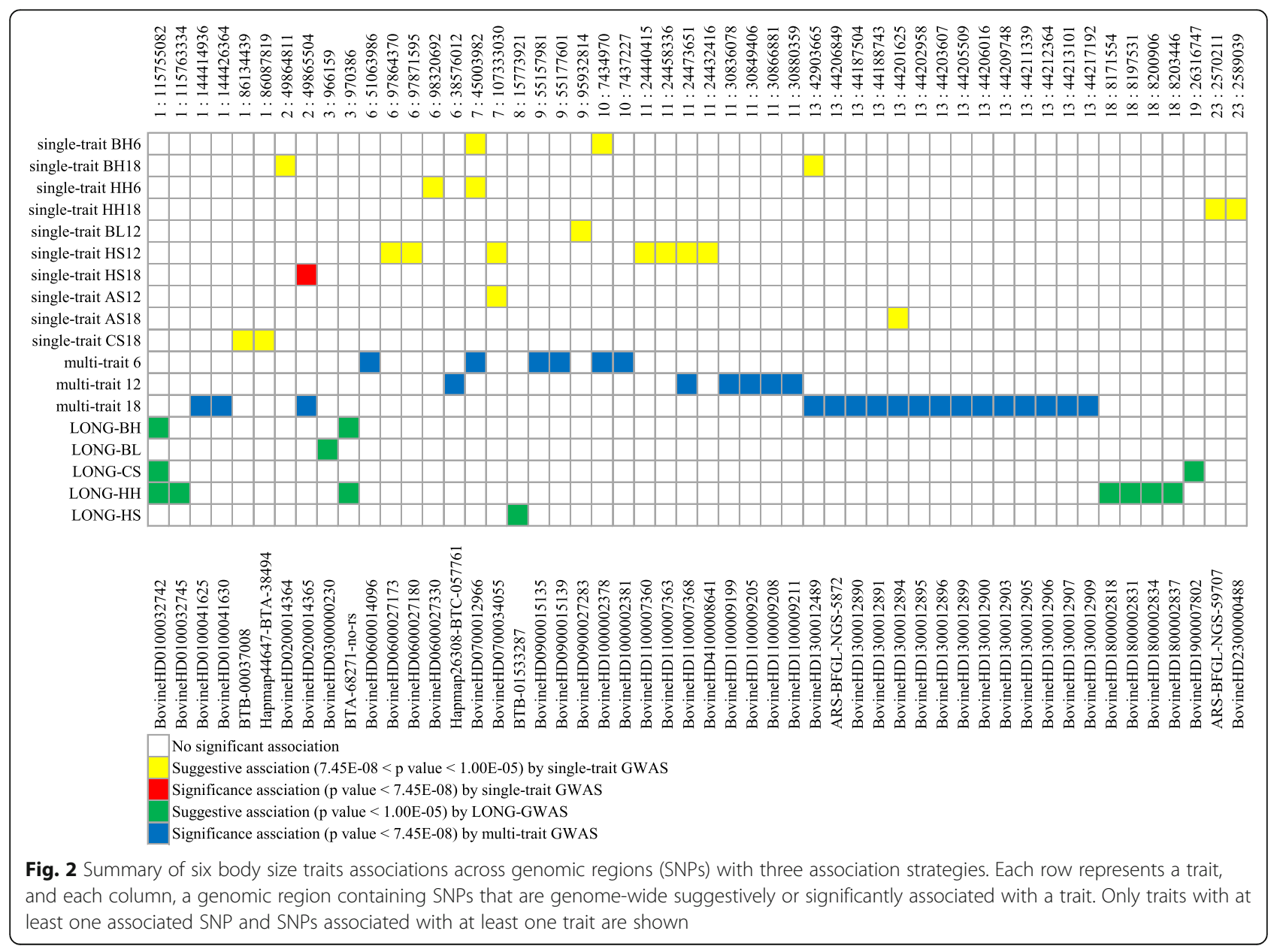


Table 1 List of suggestive candidate genes associated with six body size traits in Simmental beef cattle

\begin{tabular}{|c|c|c|c|c|c|c|c|}
\hline Genes & Related SNPS & BTA & Position (bp) & MAF & Distance (bp) & $p$ value & Associated traits \\
\hline \multirow[t]{4}{*}{ P2RY1 } & BovineHD0100032742 & 1 & $115,755,082$ & 0.40 & 79,694 & $9.51 \mathrm{E}-06$ & LONG-BH \\
\hline & BovineHD0100032742 & 1 & $115,755,082$ & 0.40 & 79,694 & $1.54 \mathrm{E}-06$ & LONG-HH \\
\hline & BovineHD0100032742 & 1 & $115,755,082$ & 0.40 & 79,694 & $9.75 \mathrm{E}-06$ & LONG-CS \\
\hline & BovineHD0100032745 & 1 & $115,763,334$ & 0.40 & 71,442 & 1.99E-06 & LONG-HH \\
\hline \multirow[t]{2}{*}{ SLC37A1 } & BovineHD0100041625 & 1 & $144,414,936$ & 0.24 & within & 7.32E-08 & multi-trait18 \\
\hline & BovineHD0100041630 & 1 & $144,426,364$ & 0.24 & within & 7.32E-08 & multi-trait18 \\
\hline \multirow[t]{2}{*}{ SOX2 } & BTB-00037008 & 1 & $86,134,439$ & 0.21 & 200,052 & 8.34E-06 & single-trait CS18 \\
\hline & Hapmap44647-BTA-38494 & 1 & $86,087,819$ & 0.19 & 153,432 & $3.58 \mathrm{E}-06$ & single-trait CS18 \\
\hline \multirow[t]{3}{*}{ SNRPD1 } & BovineHD0200014364 & 2 & $49,864,811$ & 0.20 & 128,412 & $9.22 \mathrm{E}-06$ & single-trait HS18 \\
\hline & BovineHD0200014365 & 2 & $49,865,504$ & 0.25 & 129,105 & $2.11 \mathrm{E}-08$ & single-trait HS18 \\
\hline & BovineHD0200014365 & 2 & $49,865,504$ & 0.24 & 129,105 & 4.06E-08 & multi-trait18 \\
\hline \multirow[t]{3}{*}{ MPZL1 } & BovineHD0300000230 & 3 & 966,159 & 0.38 & within & 7.60E-06 & LONG-BL \\
\hline & BTA-68271-no-rs & 3 & 970,386 & 0.42 & within & 8.93E-07 & LONG-BH \\
\hline & BTA-68271-no-rs & 3 & 970,386 & 0.42 & within & $6.64 \mathrm{E}-06$ & LONG-HH \\
\hline PCDH7 & BovineHD0600014096 & 6 & $51,063,986$ & 0.00 & 472,877 & 4.90E-08 & multi-trait6 \\
\hline \multirow[t]{3}{*}{ RASGEF1B } & BovineHD0600027173 & 6 & $97,864,370$ & 0.15 & 125,438 & 8.09E-06 & single-trait HS12 \\
\hline & BovineHD0600027180 & 6 & $97,871,595$ & 0.15 & 118,213 & 4.49E-06 & single-trait HS12 \\
\hline & BovineHD0600027330 & 6 & $98,320,692$ & 0.10 & 306,357 & $9.24 \mathrm{E}-06$ & single-trait $\mathrm{HH} 6$ \\
\hline LAP3 & Hapmap26308-BTC-057761 & 6 & $38,576,012$ & 0.39 & within & $1.94 \mathrm{E}-08$ & multi-trait12 \\
\hline \multirow[t]{3}{*}{ PTBP1 } & BovineHD0700012966 & 7 & $45,003,982$ & 0.02 & 12,339 & $3.90 \mathrm{E}-07$ & single-trait $\mathrm{HH} 6$ \\
\hline & BovineHD0700012966 & 7 & $45,003,982$ & 0.02 & 12,339 & $7.42 \mathrm{E}-06$ & single-trait $\mathrm{BH} 6$ \\
\hline & BovineHD0700012966 & 7 & $45,003,982$ & 0.02 & 12,339 & $8.23 \mathrm{E}-13$ & multi-trait6 \\
\hline \multirow[t]{2}{*}{ EFNA5 } & BovineHD0700034055 & 7 & $107,333,030$ & 0.00 & $1,716,560$ & $1.84 \mathrm{E}-06$ & single-trait HS12 \\
\hline & BovineHD0700034055 & 7 & $107,333,030$ & 0.00 & $1,716,560$ & $7.68 \mathrm{E}-06$ & single-trait AS12 \\
\hline LINGO2 & BTB-01533287 & 8 & $15,773,921$ & 0.31 & 495,444 & $7.98 \mathrm{E}-06$ & LONH-HS \\
\hline \multirow[t]{2}{*}{ MANEA } & BovineHD0900015135 & 9 & $55,157,981$ & 0.01 & within & 5.01E-08 & multi-trait6 \\
\hline & BovineHD0900015139 & 9 & $55,177,601$ & 0.01 & within & 5.01E-08 & multi-trait6 \\
\hline SNX9 & BovineHD0900027283 & 9 & $95,932,814$ & 0.23 & within & $5.18 \mathrm{E}-06$ & single-trait BL12 \\
\hline \multirow[t]{3}{*}{$\mathrm{SV} 2 \mathrm{C}$} & BovineHD1000002378 & 10 & $7,434,970$ & 0.35 & within & 4.16E-07 & single-trait $\mathrm{BH} 6$ \\
\hline & BovineHD1000002378 & 10 & $7,434,970$ & 0.35 & within & 2.99E-09 & multi-trait6 \\
\hline & BovineHD1000002381 & 10 & $7,437,227$ & 0.14 & within & $5.96 \mathrm{E}-08$ & multi-trait6 \\
\hline \multirow[t]{5}{*}{ PKDCC } & BovineHD1 100007360 & 11 & $24,440,415$ & 0.30 & 89,224 & 2.65E-06 & single-trait HS12 \\
\hline & BovineHD1 100007363 & 11 & $24,458,336$ & 0.30 & 71,303 & 4.35E-06 & single-trait HS12 \\
\hline & BovineHD1 100007368 & 11 & $24,473,651$ & 0.49 & 55,988 & $3.84 \mathrm{E}-06$ & single-trait HS12 \\
\hline & BovineHD1 100007368 & 11 & $24,473,651$ & 0.50 & 55,988 & $2.88 \mathrm{E}-08$ & multi-trait12 \\
\hline & BovineHD4100008641 & 11 & $24,432,416$ & 0.29 & 97,223 & $4.62 \mathrm{E}-06$ & single-trait HS12 \\
\hline \multirow[t]{4}{*}{ LHCGR } & BovineHD1100009199 & 11 & $30,836,078$ & 0.42 & within & 2.57E-08 & multi-trait12 \\
\hline & BovineHD1100009205 & 11 & $30,849,406$ & 0.43 & within & 4.07E-08 & multi-trait12 \\
\hline & BovineHD1 100009208 & 11 & $30,866,881$ & 0.43 & within & 4.62E-08 & multi-trait12 \\
\hline & BovineHD1 100009211 & 11 & $30,880,359$ & 0.43 & within & 4.07E-08 & multi-trait12 \\
\hline \multirow[t]{2}{*}{ SYNDIG1 } & BovineHD1300012489 & 13 & $42,903,665$ & 0.43 & 10,485 & 1.06E-06 & single-trait $\mathrm{BH} 18$ \\
\hline & BovineHD1300012489 & 13 & $42,903,665$ & 0.43 & 10,485 & $6.31 \mathrm{E}-08$ & multi-trait18 \\
\hline \multirow[t]{2}{*}{ AKR1E2 } & ARS-BFGL-NGS-5872 & 13 & $44,206,849$ & 0.49 & within & 4.17E-09 & multi-trait18 \\
\hline & BovineHD1300012890 & 13 & $44,187,504$ & 0.50 & 16,132 & $3.20 \mathrm{E}-09$ & multi-trait18 \\
\hline
\end{tabular}


Table 1 List of suggestive candidate genes associated with six body size traits in Simmental beef cattle (Continued)

\begin{tabular}{|c|c|c|c|c|c|c|c|}
\hline Genes & Related SNPs & BTA & Position (bp) & MAF & Distance (bp) & $p$ value & Associated traits \\
\hline & BovineHD1300012891 & 13 & $44,188,743$ & 0.50 & 14,893 & 4.89E-09 & multi-trait18 \\
\hline & BovineHD1300012894 & 13 & $44,201,625$ & 0.46 & 2011 & $8.08 \mathrm{E}-06$ & single-trait AS18 \\
\hline & BovineHD1300012894 & 13 & $44,201,625$ & 0.47 & 2011 & 3.84E-10 & multi-trait18 \\
\hline & BovineHD1300012895 & 13 & $44,202,958$ & 0.50 & 678 & 4.65E-08 & multi-trait18 \\
\hline & BovineHD1300012896 & 13 & $44,203,607$ & 0.50 & 29 & 6.37E-09 & multi-trait18 \\
\hline & BovineHD1300012899 & 13 & $44,205,509$ & 0.50 & within & 2.75E-09 & multi-trait18 \\
\hline & BovineHD1300012900 & 13 & $44,206,016$ & 0.49 & within & 4.18E-09 & multi-trait18 \\
\hline & BovineHD1300012903 & 13 & $44,209,748$ & 0.50 & within & 2.75E-09 & multi-trait18 \\
\hline & BovineHD1300012905 & 13 & $44,211,339$ & 0.49 & within & $2.62 \mathrm{E}-08$ & multi-trait18 \\
\hline & BovineHD1300012906 & 13 & $44,212,364$ & 0.49 & within & $3.11 \mathrm{E}-09$ & multi-trait18 \\
\hline & BovineHD1300012907 & 13 & $44,213,101$ & 0.48 & within & 5.66E-09 & multi-trait18 \\
\hline & BovineHD1300012909 & 13 & $44,217,192$ & 0.48 & 2915 & 2.63E-08 & multi-trait18 \\
\hline \multirow[t]{4}{*}{ CMIP } & BovineHD1800002818 & 18 & $8,171,554$ & 0.48 & 39,261 & $5.92 \mathrm{E}-06$ & LONG-HH \\
\hline & BovineHD1800002831 & 18 & $8,197,531$ & 0.43 & 13,284 & $6.66 \mathrm{E}-06$ & LONG-HH \\
\hline & BovineHD1800002834 & 18 & $8,200,906$ & 0.43 & 9909 & $5.72 \mathrm{E}-06$ & LONG-HH \\
\hline & BovineHD1800002837 & 18 & $8,203,446$ & 0.43 & 7369 & 4.87E-06 & LONG-HH \\
\hline WSCD1 & BovineHD1900007802 & 19 & $26,316,747$ & 0.37 & 31,164 & 5.47E-06 & LONG-CS \\
\hline \multirow[t]{2}{*}{ PRIM2 } & ARS-BFGL-NGS-59707 & 23 & $2,570,211$ & 0.36 & within & 6.65E-06 & single-trait $\mathrm{HH} 18$ \\
\hline & BovineHD2300000488 & 23 & $2,589,039$ & 0.36 & within & 8.16E-06 & single-trait $\mathrm{HH} 18$ \\
\hline
\end{tabular}

Name of trait: $B H$ body height; $B L$ body length; $H H$ hip height; $H S$ heart size; $A S$ abdominal size; $C S$ cannon bone size

Name of SNPs: Single nucleotide polymorphism name in the Bovine HD panel

BTA Bos Taurus autosome

$M A F$ minor allele frequency

Position: Position (bp) on UMD3.1

Distance: distance between SNP and the nearest gene

$P$ value: $p$-values calculated from the mixed linear model analysis

$2.11 \times 10^{-8}$ (BovineHD0200014365 for HS18), and the MAF ranging from 0.003 (BovineHD0700034055) to 0.497 (BovineHD2600012755). Among these, two SNPs near SOX2 (SRY-Box 2) on BTA1 were also identified in the liver and stomach [28]. On BTA2, two loci in the $0.69 \mathrm{~Kb}$ region were significantly associated with single-trait HS18 and one of them (BovineHD0200014365) was also associated with multi-trait18. On BTA6, three SNPs in the $0.46 \mathrm{Mb}$ region were located near RASGEF1B (RasGEF Domain Family Member 1B). On BTA7, one SNP (BovineHD0700034055) was associated with single-trait HS12 and single-trait AS12, namely EFNA5 and another SNP (BovineHD0700012966) was associated with single-trait HH6 and multi-trait6, namely PTBP1. On BTA10, one SNP (BovineHD1000002378) was associated with single-trait BH6 and multi-trait6, namely $S V 2 C$. While on BTA11, four loci in $0.04 \mathrm{Mb}$ region were associated with the single-trait HS12 and one of them (BovineHD1100007368) was also associated with multi-trait12, all of which were near PKDCC. On BTA13, SNP BovineHD1300012489 and BovineHD1300012894 were associated with single-trait $\mathrm{BH} 18$ and single-trait AS18, respectively. These two SNPs also strongly associated with multi-trait18. On BTA23, two SNPs were found within PRIM2.

\section{SNPs identified by multi-trait GWAS}

Multi-trait GWAS identified 66 SNPs within or near 36 genes that were distributed on 21 chromosomes, including 8 loci that were also identified by single-trait GWAS, which indicated that these loci suggestively regulate the development of the body growth (Table 1). Among these, two promising loci within the $11.4 \mathrm{~Kb}$ region were detected, namely SLC37A1. On BTA6, two suggestive loci were detected, one near LAP3 that associated with multi-trait12 and another near $P C D H 7$. Two genome-wide loci were identified within the $0.02 \mathrm{Mb}$ region of MANEA on BTA9. Furthermore, four promising loci were detected within in $0.04 \mathrm{Mb}$ region of LHCGR on BTA11, and 13 loci were identified within the $0.03 \mathrm{Mb}$ region of AKR1E2 on BTA13, which were also detected by single-trait analysis.

\section{SNPs identified by LONG-GWAS}

Nineteen loci were identified by LONG-GWAS, including three significant loci on 12 chromosomes (Table 1). 
Among them, two suggestive loci in the $8.3 \mathrm{~Kb}$ region near P2RY1 were detected, whereas the other (BovineHD0100032742) was also associated with LONG-CS, LONG-BH and LONG-HH. Another two loci in the 4.2 $\mathrm{Kb}$ region near MPZL1 were identified, and the latter SNP (BTA-68271-no-rs) was also associated with LONG-HH and LONG-BH. Furthermore, one suggestive SNP near LINGO2 on BTA8 was associated with LONG-HS. In addition, four promising loci in the 0.03 $\mathrm{Kb}$ region were associated with LONG-HH, namely $C M I P$, a key gene in the T-cell signaling pathway. On BTA19, a suggestive locus near WSCD1 was associated with LONG-CS. No loci were associated with AS in our research.

\section{Discussion}

We performed single-trait GWAS, multi-trait GWAS, and LONG-GWAS for six body size traits on three growth stages in Simmental beef cattle. However, the three methods yielded different results with few shared loci. The reason for this discrepancy was likely due to the restricted dataset in single-trait GWAS and LONGGWAS analyses. One universal phenomenon that cannot be ignored is that growth traits are controlled by multiple genes [29], and each method had its specific advantages in the identification of distinct loci. For example, single-trait GWAS is robust in detecting trait-specific QTLs and multi-trait GWAS is efficient for mapping pleiotropic QTLs [30], whereas LONG-GWAS can improve the detection power for time-dependent and consistent loci [31]. Thus, combining these three GWAS methods was expected to markedly improve the analysis the genetic mechanism of the body traits of beef cattle. In addition, since many complex traits have a similar architecture across diverse species [7], which prompted us to compare some of our significant genes with the previous reports that investigate the same genes and their involvements in growth. As a result, 21 suggestive genes were considered as candidate genes that were involved in the growth of cattle, swine, mice, and humans.

\section{Candidate genes identified by single-trait GWAS}

On BTA1, two SNPs were near SOX2 (SRY-Box 2), which encodes a transcription factor involved in the regulation of embryonic development $[32,33]$. The para$\log$ of this gene is SOX17, which positively affects the growth traits of cattle, and the conserved regions of this gene in human genome is closely related to body development [34]. On BTA2, two SNPs were near SNRPD1 (small nuclear ribonucleoprotein D1 polypeptide), which is a member of the ghrelin receptor family, and the encoded protein is involved in zinc-dependent signaling in epithelial tissue [35]. On BTA6, variations near RASGEF1B (RasGEF domain family member 1B) were associated with body height [36]. In addition, body height was positively correlated with calcium absorption, which is an important determinant of calcium balance [37]. On BTA7, a SNP near EFNA5 (ephrin A5) was associated with two traits (HS and AS) at the same stage. It was also identified as a candidate gene for growth traits in broiler chicken [38]. Another SNP near PTBP1 (polypyrimidine tract binding protein 1 ) was found to show genome-wide association with growth traits at 6 months by both single-trait and multi-trait GWAS. Its expression level determined the release of insulin, thereby affecting development [39]. On BTA9, a SNP (BovineHD0900027283) located in SNX9 (sorting nexin 9), as olfactory receptor, was associated with growth traits in Yorkshire pigs [40]. The SNP near SV2C (synaptic vesicle glycoprotein $2 \mathrm{C}$ ) was associated with $\mathrm{BH}$ by both single-trait and multi-trait GWAS. This gene was reported to modulate dopamine release in neural and endocrine cells [41]. On BTA11, PKDCC (protein kinase domain containing, cytoplasmic) was associated with HS in both single-trait and multi-trait analyses. This gene was involved in the maintenance of bone density in humans [42]. On BTA13, SYNDIG1 (synapse differentiation inducing 1 ) has been reported as a factor influencing the final weight and backfat thickness of Landrace pigs [43], whereas the AKR1E2 (aldo-keto reductase family 1 member E2) variant was associated with body length and girth in cattle [44]. On BTA23, the PRIM2 (DNA primase subunit 2) was associated with body weight and trait changes in pigs $[45,46]$.

\section{Single-trait GWAS versus multi-trait GWAS}

Multiple-trait analysis of linkage experiments has been reported to significantly enhance the power to detect common SNPs across traits $[47,48]$. Therefore, we used multi-trait analysis to complement single-trait GWAS rather than to replace it. In the single-trait GWAS, the minimum $p$ values for the three stages were $3.90 \mathrm{E}-07$, 9.92E-07 and 2.11E-08 respectively. These three values decreased to $8.23 \mathrm{E}-13,1.73 \mathrm{E}-09$ and $3.84 \mathrm{E}-10$ in the multi-trait GWAS, respectively. We also identified several critical loci as follows. On BTA1, the SLC37A1 (solute carrier family 37 , member A1) gene, which encodes a glucose-6-phosphate transporter that is involved in the homeostasis of blood glucose [48], was found to be the best candidate gene for modifying milk production traits [49]. On BTA6, LAP3 (leucine aminopeptidase 3 ) was reported to play critical roles in the regulation of hormone levels and protein maturation. Another study demonstrated that putative regulatory elements in the $\mathrm{PCDH7}$ (protocadherin 7) gene may have roles in residual feed intake in Nelore cattle [50]. In addition, MANEA (mannosidase endo-alpha), which has roles in proteolysis, was associated with the birth weight of 
Canchim beef cattle [17]. On BTA11, a mutation in the LHCGR (luteinizing hormone/choriogonadotropin receptor) gene was as the cause of empty follicle syndrome [51].

\section{Single-trait GWAS versus LONG-GWAS}

We used LONG-GWAS, which involved multiple phenotype measurements for each individual [24]. One disadvantage of this method was that incorporating all data may have overwhelmed significant signal, that is, if QTL effects varied during the different stages [52]. In this study, these time-specific expressed QTLs identified by the single-trait and multi-trait GWAS were not detected by LONG-GWAS. However LONG-GWAS also detected some significant functional loci as follows. On BTA1, P2RY1 (purinergic receptor P2Y1), a candidate gene that affects the serum $\mathrm{Ca}^{2+}$, encoded for a member of the family of $\mathrm{G}$ protein-coupled receptor family that works as receptor for extracellular ATP and ADP [53]. On BTA3, the MPZL1 (myelin protein zero like 1) gene could significantly enhance the migratory and metastatic potential of hepatocellular carcinoma cells by phosphorylating and activating the pro-metastatic protein [54]. Besides on BTA8, LINGO2 (leucine rich repeat and Ig domain containing 2), which is expressed in the central nervous system of mouse embryos, has been reported to associate with the body mass in a cohort of elderly Swedes [55]. On BTA18, CMIP (C-Maf inducing protein), a candidate gene for reading-related traits, was also associated with plasma lipoprotein levels [56]. Moreover, WSCD1 (WSC domain containing 1), which encodes a protein with sulfotransferase activity that participates in the metabolism of glucose, was a candidate gene for feed efficiency and feeding behaviors in the White Duroc $x$ Erhualian F2 population [57].

\section{Conclusions}

In conclusion, a total of 58 SNPs corresponding to 21 genes were found to be associated with six body size traits at 6,12 and 18 months. Future studies characterizing the functions of these candidate genes may uncover the genetic architecture underlying the body size traits in Simmental beef cattle.

\section{Methods}

\section{Resource population and phenotypes collection}

Simmental beef cattle (born between 2008 and 2015) were established in Ulgai, Xilingole League, Inner Mongolia of China. Six body size traits at three growth stages $(6,12$, and 18 months after birth) were measured simultaneously for each individual. The details of trait measurement are as follows: $\mathrm{BH}$, also named wither height, the height from wither to ground; BL, the length from the front edge of the scapula to the back edge of the ischial tuberosity; $\mathrm{HH}$, height from hip to ground;
HS, also named heart girth, the chest girth in the back edge of scapula; AS, the girth of the thickest part of the abdomen; CS, the girth of the thinnest cannon bone. In addition, only cattle (133 individuals, Table S1) whose phenotype records overlapped during three growth stages were used for LONG-GWAS analysis. The blood sample specimens were collected during the regular quarantine inspection of the farms was conducted. After this study, all individuals were slaughtered in strict compliance with the Institutional Meat Purchase Specifications for fresh beef. All procedures were conducted in strict compliance with the guidelines established by the Ministry of Agriculture of China. Some descriptive statistics and heritability estimates of six traits at three growth stages are presented in Table 2.

\section{Genotyping and quality control}

Genomic DNA was isolated from blood samples using the TIANamp Blood DNA Kit (Tiangen Biotech Co.Ltd., Beijing, China). DNA quality was acceptable when the A260/A280 radio was between 1.8 and 2.0. Genotyping was performed with the Illumina BovineHD Beadchip (Illumina Inc., San Diego, CA, USA) and the PLINK v1.07 Software was used for quality control [58]. In this study, animals with a call rate $(<0.9)$ were discarded. SNPs were deleted the following standards, including minor allele frequency $(<0.01)$, SNP call rate $(<0.05)$

Table 2 Descriptive statistics of body size traits at 6, 12, and 18 months after birth

\begin{tabular}{|c|c|c|c|c|c|c|c|}
\hline Month & Trait (cm) & $\mathrm{N}^{\mathrm{a}}$ & Mean & Min. & Max. & SD & $h^{2}$ (SE) \\
\hline \multirow[t]{6}{*}{6} & $\mathrm{BH}$ & 218 & 100.44 & 80 & 127 & 9.803 & $0.49 \pm 0.04$ \\
\hline & $\mathrm{HH}$ & 121 & 105.01 & 85 & 136 & 10.42 & $0.51 \pm 0.06$ \\
\hline & $B L$ & 214 & 105.31 & 72 & 138 & 10.65 & $0.52 \pm 0.05$ \\
\hline & $\mathrm{HS}$ & 213 & 125.44 & 89 & 170 & 15.61 & $0.51 \pm 0.06$ \\
\hline & AS & 211 & 140.87 & 97 & 188 & 17.54 & $0.51 \pm 0.07$ \\
\hline & CS & 119 & 16.377 & 12 & 21.5 & 2.323 & $0.62 \pm 0.04$ \\
\hline \multirow[t]{6}{*}{12} & $\mathrm{BH}$ & 457 & 116.69 & 97 & 135 & 7.274 & $0.29 \pm 0.08$ \\
\hline & $\mathrm{HH}$ & 453 & 123.69 & 105 & 142 & 7.415 & $0.27 \pm 0.08$ \\
\hline & $B L$ & 453 & 130.18 & 104 & 157 & 9.541 & $0.53 \pm 0.07$ \\
\hline & $\mathrm{HS}$ & 454 & 168.24 & 129 & 202 & 13.09 & $0.33 \pm 0.05$ \\
\hline & AS & 454 & 198.53 & 155 & 238 & 15.24 & $0.30 \pm 0.07$ \\
\hline & CS & 436 & 18.069 & 15 & 21.5 & 1.221 & $0.29 \pm 0.06$ \\
\hline \multirow[t]{6}{*}{18} & $\mathrm{BH}$ & 516 & 126.46 & 105 & 139 & 4.577 & $0.28 \pm 0.06$ \\
\hline & $\mathrm{HH}$ & 267 & 132.61 & 109 & 147 & 5.453 & $0.41 \pm 0.04$ \\
\hline & $B L$ & 514 & 144.19 & 123 & 169 & 7.843 & $0.28 \pm 0.07$ \\
\hline & $\mathrm{HS}$ & 513 & 188.11 & 160 & 214 & 8.133 & $0.30 \pm 0.08$ \\
\hline & AS & 512 & 219.01 & 193 & 244 & 8.909 & $0.14 \pm 0.06$ \\
\hline & CS & 381 & 20.155 & 17 & 23 & 1.506 & $0.54 \pm 0.07$ \\
\hline
\end{tabular}

$h^{2}$ heritability, SE standard error, $B H$ body height, $B L$ body length, $H H$ hip height, HS heart size, $A S$ abdominal size, CS cannon bone size

${ }^{\mathrm{a}}$ Number of animal with phenotypes 
and Hardy-Weinberg equilibrium values $\left(p<1 \times 10^{-6}\right)$. Finally, 671,192 SNPs on 29 autosomal chromosomes with an average distance of $3 \mathrm{~kb}$ were generated for the analysis.

\section{Single-trait GWAS}

For six body size traits in three growth stage, we performed single-trait GWAS, respectively. The compressed mixed linear model (CMLM) was called because it reduced computing time by clustering individuals into groups, increased the power in QTN detection by eliminating the need to re-compute variance components, and enhanced the effectiveness in correcting the inflation from the polygenic background and controlling the bias of population stratification $[59,60]$. Briefly, a principal components analysis (PCA) was performed and a kinship matrix was calculated using the Genome Association and Prediction Integrated Tool (GAPIT) package in R v3.4.2 [61]. To revise the effects of population structure, the $\mathbf{Q}$ matrix was reflected by the PCA. To replace the incomplete pedigrees, the $\mathbf{K}$ matrix was calculated by the VanRaden algorithm [62]. This model is as follows:

$$
\mathrm{y}=\mathrm{Wu}+\mathrm{X} \beta+\mathrm{Zu}+\mathrm{e}
$$

where $y$ is a vector of the observed phenotypes; $\boldsymbol{W}$ was a vector of SNP genotype indicators, which was coded as 0,1 and 2 corresponding to the three genotypes $\mathrm{AA}, \mathrm{AB}$, and $\mathrm{BB}$ with $\mathrm{B}$ being the minor allele. $v$ was the effect of marker, which is treated as a fixed effect; Variable $\mathbf{X}$ is an incidence matrix for non-genetic fixed effects, and $\boldsymbol{\beta}$ is a non-genetic vector of fixed effects including month ages (time of birth to measurement), enter weight (weight of just entering the farm), fattening days (time of entering farm to measurement) and principal component effects (the top three eigenvectors of the $\mathbf{Q}$ matrix). Variable $\mathbf{Z}$ is an incidence matrix for a vector of polygenic effects, and parameter $\boldsymbol{u}$ is a vector for residual polygenic effects with an assumed $N\left(0, K \sigma^{2}\right)$ distribution, where $\sigma^{2}$ is the polygenic variance and $K$ is a marker inferred kinship matrix. While $\boldsymbol{e}$ is a vector for random residual errors with a putative $N\left(0, I \sigma_{e}^{2}\right)$ distribution, where $\sigma_{e}^{2}$ is the residual variance. The heritability $\left(h^{2}\right)$ is defined as: $h^{2}=\frac{\sigma^{2}}{\sigma^{2}+\sigma_{e}^{2}}$. The CMLM analysis was performed with GAPIT Software package (http://www. maizegenetics.net/gapit). Quantile-quantile (Q-Q) plots were generated to visualize the goodness of fitting for the GWAS model accounted by the population structure and familial relatedness. The negative logarithm of the $p$ value from the model was calculated against the expected value based on the null hypothesis. The threshold p value after Bonferroni correction was $0.05 / N=7.45 \times$ $10^{-8}$, where $\mathrm{N}$ is the number of SNPs. In light of the fact that the Bonferroni correction results were too stringent with low statistical power [63]. Hence, we adopted the false discovery rate (FDR) to determine the threshold values for Single-GWAS, Multi-trait GWAS and LONGGWAS. The FDR was set as 0.01 , and the threshold $\mathrm{p}$ value was calculated as follows:

$$
P=\mathrm{FDR} \times n / m
$$

where $n$ is the number of $P<0.01$ in the results, and $m$ is the total number of SNPs [64].

\section{Multi-trait GWAS}

For the three growth stages, the multi-trait GWAS were conducted to detect pleiotropic SNPs and the model was a Chi square statistic, which approximately followed a Chi square distribution with the number of traits tested as the number of degrees of freedom. It was calculated for each SNP using the following formula [65]:

$$
\begin{aligned}
& t_{i}=\frac{\left|\widehat{v}_{i}\right|}{\sqrt{V\left(\widehat{v_{i}}\right)}} \\
& \chi 2 \text { multi-trait }=t_{i}^{\prime} \mathrm{V}^{-1} t_{i} .
\end{aligned}
$$

where $\widehat{v}_{i}$ is the estimate of $\boldsymbol{v}$ and the corresponding variance $V\left(\widehat{v}_{i}\right)$ can be obtained by the compressed mixed linear model (CMLM); While $t_{i}$ is the $6 \times 1$ vector of the signed $\mathrm{t}$-values of the $i$ th SNP from the abovementioned single-trait GWAS for the six traits. Matrix $t_{i}^{\prime}$ is the transpose of the vector $t_{i}$, and $V^{-1}$ is the inverse of the $6 \times 6$ correlation matrix between traits, which was calculated by the estimated effects of the qualified SNPs (signed $t$ values).

\section{Long-GWAS}

For these individuals that completely obtained body size information at three growth stages, we conducted a longitudinal GWAS by LONG-GWAS [24]. This model was similar to CMLM, except that the phenotypic variance was partitioned to SNPs, fixed factors (the abovementioned $\beta$ vector), polygenic effects, time stage effects and residual variance. Moreover, numerous studies have reported that the longitudinal design could facilitate the identification of time-dependent and consistent loci, which increased the statistical power due to their effectiveness in incorporating the correlation structure of multiple measurements and alleviating the multiple testing burden $[26,27,66]$. The code data implementing this method may be found at http://genetics.cs.ucla.edu/longGWAS/. This model is as follows:

$$
\mathrm{y}^{*}=\mathrm{Wv}+\mathrm{Zu}+\mathrm{y}+\mathrm{e}
$$

In this formula, $y^{*}$ is the adjusted phenotype (these fixed effects mentioned in CMLM were adjusted in order to account for additional confounding). The 
incidence matrix $W, Z$, the vector $\boldsymbol{v}, \boldsymbol{u}$ and $\boldsymbol{e}$ are consistent with CMLM mentioned above. Differently, the parameter $\gamma$ is a vector for time stage effects with a putative $N\left(0, \sigma_{v}^{2} \boldsymbol{D}\right)$ distribution, where $\mathbf{D}$ is a known block diagonal matrix representing the covariance between permanent environmental components. The $\mathbf{D}$ matrix was calculated by this formula: $\mathbf{D}=\mathbf{E} \otimes \mathbf{I}$, where $\mathbf{E}$ is a $3 \times 3$ matrix representing the covariance between the set of 3 time points for each individual.

\section{Identification and annotation of candidate genes}

The UMD3.1 genome assembly was used to located genes for annotation, and the QTLdb database (http:// www.animalgenome.org) was applied to search for related QTL regions.

\section{Supplementary information}

Supplementary information accompanies this paper at https://doi.org/10. 1186/s12863-020-0837-6.

Additional file 1: Figure S1. The strengths of genome-wide association studies (GWAS) are illustrated by the Manhattan plots on the left panel. The deviations of the signals from null hypothesis are illustrated as the Quantile-Quantile (QQ) plots on the right panel. The negative logarithms of the observed (y axis) and the expected ( $x$ axis) $P$ values are plotted for each SNP (dot). GWAS were performed six body size traits months 6, 12 and 18 after birth separately. Each analysis is labeled as trait $(\mathrm{BH}$ or $\mathrm{HH})$ and month on the far right. The number neighboring each trait indicates the age of measurement (e.g., $\mathrm{BH} 6=$ Body Height at 6 months). The 29 chromosomes are color coded.

Additional file 2: Figure S2. The strengths of genome-wide association studies (GWAS) are illustrated by the Manhattan plots on the left panel. The deviations of the signals from null hypothesis are illustrated as the Quantile-Quantile (QQ) plots on the right panel. The negative logarithms of the observed ( $y$ axis) and the expected ( $x$ axis) $P$ values are plotted for each SNP (dot). GWAS were performed six body size traits months 6, 12 and 18 after birth separately. Each analysis is labeled as trait $(\mathrm{BH}$ or $\mathrm{HH})$ and month on the far right. The number neighboring each trait indicates the age of measurement (e.g., $\mathrm{BH} 6=$ Body Height at 6 months). The 29 chromosomes are color coded.

Additional file 3: Table S1. Descriptive statistics of 133 phenotypic records in LONG-GWAS method.

\section{Abbreviations}

GWAS: Genome-wide association study; BH: Body height; BL: Body length; $\mathrm{HH}$ : Hip height; HS: Heart size; AS: Abdominal size; CS: Cannon bone size; SNP: Single nucleotide polymorphism; QTL: Quantitative trait loci;"; PCA: Principal components analysis; CMLM: Compressed mixed linear model;" GAPIT: Genome Association and Prediction Integrated Tool; BTA: Bos Taurus chromosome; LONG-GWAS: Longitudinal GWAS

\section{Acknowledgements}

Not applicable.

\section{Authors' contributions}

BA wrote, and $\mathrm{J}$ and $\mathrm{HG}$ revised the paper. $\mathrm{LX} 1$ and $J X$ performed experiments. MS, $\mathrm{JN}$, and LZ collected the GWAS data. XW, JM, TC, and LX2 interpreted the data. All authors reviewed and approved the final manuscript

\section{Funding}

This work was supported by funds from the National Natural Science Foundations of China (NSFC 31872975, 31472079 and 31702084) and Cattle Breeding Innovative Research Team (cxgc-ias-03). NSFC funded the collection, analysis, and interpretation of data. Cattle Breeding Innovative Research Team supported statistical analysis and writing of paper.

\section{Availability of data and materials}

We confirm that all raw data underlying our findings are publicly available without restriction. Data is available from the Dryad Digital Repository: doi:https://doi.org/10.5061/dryad.4qc06.

\section{Ethics approval and consent to participate}

All animals used in the study were treated following the guidelines established by the Council of China Animal Welfare. Protocols of the experiments were approved by the Science Research Department of the Institute of Animal Sciences, Chinese Academy of Agricultural Sciences (CAAS), Beijing, China (approval number: RNL09/07). The use of animals and private land in this study was approved in written by their respective legal owners.

\section{Consent for publication}

Not applicable.

\section{Competing interests}

The authors declare that they have no competing interests.

\section{Author details}

Institute of Animal Science, Chinese Academy of Agricultural Science, Beijing 100193, China. ${ }^{2}$ Institute of Basic Medical Sciences, Westlake Institute for Advanced Study, Hangzhou 310000, China. ${ }^{3}$ Zhuang Yuan Veterinary Station of Qixia city, Yantai 265300, China. ${ }^{4}$ Heibei Livestock Breeding Workstation, Shijiazhuang 050061, China.

Received: 12 August 2019 Accepted: 4 March 2020

Published online: 14 March 2020

\section{References}

1. Heinrichs AJ, Losinger WC. Growth of Holstein dairy heifers in the United States. J Anim Sci. 1998;76:1254-60.

2. Heinrichs AJ, Erb HN, Rogers GW, Cooper JB, Jones CM. Variability in Holstein heifer heart-girth measurements and comparison of prediction equations for live weight. Prev Vet Med. 2007;78:333-8.

3. Lund T, Miglior F, Dekkers JCM, Burnside EB. Genetic relationships between clinical mastitis, somatic cell count, and udder conformation in Danish Holsteins. Livest Prod Sci. 1994:39:243-51.

4. Vollema AR, Van Der Beek S, Harbers AGF, De Jong G. Genetic evaluation for longevity of Dutch dairy bulls. J Dairy Sci. 2000:83:2629-39.

5. Wu X, Fang M, Liu L, Wang S, Liu J, Ding X, et al. Genome wide association studies for body conformation traits in the Chinese Holstein cattle population. BMC Genomics. 2013;14:897.

6. Xia J, Oi X, Wu Y, Zhu B, Xu L, Zhang L, et al. Genome-wide association study identifies loci and candidate genes for meat quality traits in Simmental beef cattle. Mamm Genome. 2016;27:246-55.

7. Pryce JE, Hayes BJ, Bolormaa S, Goddard ME. Polymorphic regions affecting human height also control stature in cattle. Genetics. 2011;187:981-4.

8. Silventoinen K, Magnusson PKE, Tynelius P, Kaprio J, Rasmussen F. Heritability of body size and muscle strength in young adulthood: a study of one million Swedish men. Genet Epidemiol. 2008:32:341-9.

9. Zhang X, Chu Q, Guo G, Dong G, Li X, Zhang Q, et al. Genome-wide association studies identified multiple genetic loci for body size at four growth stages in Chinese Holstein cattle. PLoS One. 2017;12:e0175971.

10. Bouwman AC, Daetwyler HD, Chamberlain AJ, Ponce $C H$, Sargolzaei M, Schenkel FS, et al. Meta-analysis of genome-wide association studies for cattle stature identifies common genes that regulate body size in mammals. Nat Genet. 2018;50:362-67.

11. Lesosky M, Dumas S, Conradie I, Handel IG, Jennings A, Thumbi S, et al. A live weight-heart girth relationship for accurate dosing of east African shorthorn zebu cattle. Trop Anim Health Prod. 2012;45:311-6.

12. Dechow CD, Rogers GW, Klei L, Lawlor TJ. Heritabilities and correlations among body condition score, dairy form and selected linear type traits. J Dairy Sci. 2010:86:2236-42.

13. Bardakcioglu HE, Sekkin S, Toplu HDO. Relationship between some teat and body measurements of Holstein cows and sub-clinical mastitis and milk yield. J Anim Vet Adv. 2011;10:1735-7. 
14. Jiang L, Liu J, Sun D, Ma P, Ding X, Yu Y, et al. Genome wide association studies for milk production traits in Chinese Holstein population. PLoS One. 2010;5:e13661

15. Meredith BK, Kearney FJ, Finlay EK, Bradley DG, Fahey AG, Berry DP, et al. Genome-wide associations for milk production and somatic cell score in Holstein-Friesian cattle in Ireland. BMC Genet. 2012;13:21.

16. Cole JB, Wiggans GR, Ma L, Sonstegard TS, Lawlor TJ, Crooker BA, et al. Genome-wide association analysis of thirty one production, health, reproduction and body conformation traits in contemporary U.S. Holstein cows. BMC Genomics. 2011;12:408.

17. Buzanskas ME, Grossi DA, Ventura RV, Schenkel FS, Sargolzaei M, Meirelles SLC, et al. Genome-wide association for growth traits in canchim beef cattle. PLoS One. 2014;9:e94802.

18. Jin B, Bao WJ, Wu ZQ, Xia XH. In situ monitoring of protein adsorption on a nanoparticulated gold film by attenuated total reflection surface-enhanced infrared absorption spectroscopy. Langmuir. 2012;28:9460-5.

19. Huang W, Kirkpatrick BW, Rosa GJM, Khatib H. A genome-wide association study using selective DNA pooling identifies candidate markers for fertility in Holstein cattle. Anim Genet. 2010;41:570-8.

20. Sahana G, Guldbrandtsen B, Bendixen C, Lund MS. Genome-wide association mapping for female fertility traits in Danish and Swedish Holstein cattle. Anim Genet. 2010;41:579-88.

21. Sorbolini S, Bongiorni S, Cellesi M, Gaspa G, Dimauro C, Valentini A, et al. Genome wide association study on beef production traits in Marchigiana cattle breed. J Anim Breed Genet. 2017;134:43-8.

22. Santana MHA, Utsunomiya YT, Neves HHR, Gomes RC, Garcia JF, Fukumasu $H$, et al. Genome-wide association analysis of feed intake and residual feed intake in Nellore cattle. BMC Genet. 2014;15.

23. Jahuey-Martínez FJ, Parra-Bracamonte GM, Sifuentes-Rincón AM, MartínezGonzález JC, Gondro C, García-Pérez CA, et al. Genomewide association analysis of growth traits in charolais beef cattle. J Anim Sci. 2016;94:4570-82.

24. Furlotte NA, Eskin E, Eyheramendy S. Genome-wide association mapping with longitudinal data. Genet Epidemiol. 2012;36:463-71.

25. Porter HF, O'Reilly PF. Multivariate simulation framework reveals performance of multi-trait GWAS methods. Sci Rep. 2017;7:38837.

26. Das K, Li J, Wang Z, Tong C, Fu G, Li Y, et al. A dynamic model for genomewide association studies. Hum Genet. 2011;129:629-39.

27. Kim S, Xing EP. Statistical estimation of correlated genome associations to a quantitative trait network. PLoS Genet. 2009;5:e1000587.

28. Arnold K, Sarkar A, Yram MA, Polo JM, Bronson R, Sengupta S, et al. Sox2 + adult stem and progenitor cells are important for tissue regeneration and survival of mice. Cell Stem Cell. 2011:9:317-29.

29. Dekkers JCM. Commercial application of marker- and gene-assisted selection in livestock: Strategies and lessons1,2. J Anim Sci. 2004;82(suppl_ 13):E313-28. https://doi.org/10.2527/2004.8213_supplE313x.

30. Guo Y, Huang Y, Hou L, Ma J, Chen C, Ai H, et al. Genome-wide detection of genetic markers associated with growth and fatness in four pig populations using four approaches. Genet Sel Evol. 2017:49:21.

31. Yi G, Shen M, Yuan J, Sun C, Duan Z, Qu L, et al. Genome-wide association study dissects genetic architecture underlying longitudinal egg weights in chickens. BMC Genomics. 2015;16:746.

32. Chen S, Li X, Lu D, Xu Y, Mou W, Wang L, et al. SOX2 regulates apoptosis through MAP 4K4-Survivin signaling pathway in human lung cancer cells. Carcinog. 2014;35:613-23.

33. Tani $Y$, Akiyama $Y$, Fukamachi $H$, Yanagihara K, Yuasa $Y$. Transcription factor SOX2 up-regulates stomach-specific pepsinogen a gene expression. J Cancer Res Clin Oncol. 2007;133:263-9.

34. Pausch H, Flisikowski K, Jung S, Emmerling R, Edel C, Götz KU, et al. Genome-wide association study identifies two major loci affecting calving ease and growth-related traits in cattle. Genetics. 2011;187:289-97.

35. Luechtefeld R. A general-purpose collaborative system: Theory and design of the dialogic web. 2017. IEEE Technol Eng Manag Soc Conf TEMSCON. 2017;2017:455-9.

36. He M, Xu M, Zhang B, Liang J, Chen $P$, Lee JY, et al. Meta-analysis of genome-wide association studies of adult height in east Asians identifies 17 novel loci. Hum Mol Genet. 2015;24:1791-800.

37. Lundeen EA, Stein AD, Adair LS, Behrman JR, Bhargava SK, Dearden KA, et al. Height-for-age z scores increase despite increasing height deficits among children in 5 developing countries. Am J Clin Nutr. 2014;100:821-5.

38. Liu $R$, Sun $Y$, Zhao $G$, Wang $H$, Zheng $M$, Li $P$, et al. Identification of loci and genes for growth related traits from a genome-wide association study in a slow- $\times$ fast-growing broiler chicken cross. Genes Genomics. 2015;37:829-36.

39. Hansen $T H$, Vestergaard $H$, Jørgensen $T$, Jørgensen ME, Lauritzen $T$, Brandslund I, et al. Impact of PTBP1 rs11085226 on glucose-stimulated insulin release in adult Danes. BMC Med Genet. 2015;16:17.

40. Meng Q, Wang K, Liu X, Zhou H, Xu L, Wang Z, et al. Identification of growth trait related genes in a Yorkshire purebred pig population by genome-wide association studies. Asian Australasian J Anim Sci. 2017:30:462-9.

41. Dunn AR, Stout KA, Ozawa M, Lohr KM, Hoffman CA, Bernstein Al, et al. Synaptic vesicle glycoprotein 2C (SV2C) modulates dopamine release and is disrupted in Parkinson disease. Proc Natl Acad Sci U S A. 2017;114:E2253-62.

42. Zhou H, Mori S, Ishizaki T, Takahashi A, Matsuda K, Koretsune Y, et al. Genetic risk score based on the prevalence of vertebral fracture in Japanese women with osteoporosis. Bone Reports. 2016;5:168-72. https://doi.org/10. 1016/j.bonr.2016.07.001.

43. Lee YS, Shin D, Song KD. Dominance effects of ion transport and ion transport regulator genes on the final weight and backfat thickness of landrace pigs by dominance deviation analysis. Genes Genomics. 2018;40: 1331-8.

44. Du M, Auer PL, Jiao S, Haessler J, Altshuler D, Boerwinkle E, et al. Wholeexome imputation of sequence variants identified two novel alleles associated with adult body height in African Americans. Hum Mol Genet. 2014;23:6607-15.

45. Borowska A, Reyer H, Wimmers K, Varley PF, Szwaczkowski T. Detection of pig genome regions determining production traits using an information theory approach. Livest Sci. 2017;205:31-5.

46. Wang L, Zhang L, Yan H, Liu X, Li N, Liang J, et al. Genome-wide association studies identify the loci for 5 exterior traits in a large white $x$ Minzhu pig population. PLoS One. 2014;9:e103766.

47. Klomp HM, Steyerberg EW, Ubbink DT. Letter: systematic review and metaanalysis of controlled trials assessing spinal cord stimulation for inoperable critical leg ischaemia (Br J Surg 2004; 91: 948-955) [2] (multiple letters). Br J Surg. 2005;92:120-1.

48. Andersson L, Haley CS, Ellegren $H$, Knott SA, Johansson M, Andersson $K$, et al. Genetic mapping of quantitative trait loci for growth and fatness in pigs. Science. 1994;263:1771-4.

49. Kemper KE, Littlejohn MD, Lopdell T, Hayes BJ, Bennett LE, Williams RP, et al. Leveraging genetically simple traits to identify small-effect variants for complex phenotypes. BMC Genomics. 2016;17:858.

50. Jung EJ, Park HB, Lee JB, Yoo CK, Kim BM, Kim HI, et al. Genome-wide association study identifies quantitative trait loci affecting hematological traits in an F2intercross between landrace and Korean native pigs. Anim Genet. 2014:45:534-41.

51. Yariz KO, Walsh T, Uzak A, Spiliopoulos M, Duman D, Onalan G, et al. Inherited mutation of the luteinizing hormone/choriogonadotropin receptor (LHCGR) in empty follicle syndrome. Fertil Steril. 2011;96:e125-30

52. Zhang Z, Hong Y, Gao J, Xiao S, Ma J, Zhang W, et al. Genome-wide association study reveals constant and specific loci for hematological traits at three time stages in a white Duroc $\times$ Erhualian $\mathrm{F} 2$ resource population. PLoS One. 2013;8:e63665.

53. Nishi $H_{1}$ Arai H, Momiyama T. NCl-H295R, a human adrenal cortex-derived cell line, expresses Purinergic receptors linked to Ca2+-mobilization/influx and cortisol secretion. PLoS One. 2013:8:e71022.

54. Jia D, Jing Y, Zhang Z, Liu L, Ding J, Zhao F, et al. Amplification of MPZL1/ PZR promotes tumor cell migration through Src-mediated phosphorylation of cortactin in hepatocellular carcinoma. Cell Res. 2014;24:204-17.

55. Rask-Andersen M, Almén MS ällma., Lind L, Schiöth HB. Association of the LINGO2-related SNP rs 10968576 with body mass in a cohort of elderly Swedes. Mol Genet Genomics. 2015;290:1485-1491.

56. Bryant EK, Dressen AS, Bunker CH, Hokanson JE, Hamman RF, Kamboh MI, et al. A multiethnic replication study of plasma lipoprotein levels-associated SNPs identified in recent GWAS. PLoS One. 2013:8:e63469.

57. Guo YM, Zhang ZY, Ma JW, Ai HS, Ren J, Huang LS. A genomewide association study of feed efficiency and feeding behaviors at two fattening stages in a white duroc $\times$ erhualian F2 population. J Anim Sci. 2015;93:1481-9.

58. Purcell S, Neale B, Todd-Brown K, Thomas L, Ferreira MAR, Bender D, et al. PLINK: a tool set for whole-genome association and population-based linkage analyses. Am J Hum Genet. 2007:81:559-75. https://doi.org/10.1086/519795.

59. Wang SB, Feng JY, Ren WL, Huang B, Zhou L, Wen YJ, et al. Improving power and accuracy of genome-wide association studies via a multi-locus mixed linear model methodology. Sci Rep. 2016;6:19444 
60. Zhang Z, Ersoz E, Lai CQ, Todhunter RJ, Tiwari HK, Gore MA, et al. Mixed linear model approach adapted for genome-wide association studies. Nat Genet. 2010;42:355-60.

61. Lipka AE, Tian F, Wang Q, Peiffer J, Li M, Bradbury PJ, et al. GAPIT: genome association and prediction integrated tool. Bioinformatics. 2012;28:2397-9.

62. VanRaden PM. Efficient methods to compute genomic predictions. J Dairy Sci. 2008:91:4414-23.

63. Nakagawa S. A farewell to Bonferroni: the problems of low statistical power and publication bias. Behav Ecol. 2004;15:1044-5.

64. Benjamini $Y$, Hochberg $Y$. Controlling the false discovery rate: a practical and powerful approach to multiple testing. J R Stat Soc Ser B. 1995;57:289-300.

65. Bolormaa S, Porto Neto LR, Zhang YD, Bunch RJ, Harrison BE, Goddard ME, et al. A genome-wide association study of meat and carcass traits in australian cattle. J Anim Sci. 2011;89:2297-309.

66. Zhou X, Stephens M. Efficient multivariate linear mixed model algorithms for genome-wide association studies. Nat Methods. 2014;11:407-9.

\section{Publisher's Note}

Springer Nature remains neutral with regard to jurisdictional claims in published maps and institutional affiliations.

Ready to submit your research? Choose BMC and benefit from:

- fast, convenient online submission

- thorough peer review by experienced researchers in your field

- rapid publication on acceptance

- support for research data, including large and complex data types

- gold Open Access which fosters wider collaboration and increased citations

- maximum visibility for your research: over $100 \mathrm{M}$ website views per year

At $\mathrm{BMC}$, research is always in progress.

Learn more biomedcentral.com/submissions 